\title{
COMMENT
}

\section{No stone unturned}

\author{
Gregory A Petsko*
}

Last month, I wrote about what I consider to be one of the most serious problems facing the life sciences in the age of genomics: the increasing polarization between those who do what they call 'basic research' and those who do what is termed 'translational research' [http:// genomebiology.com/2010/11/2/107]. I argued that we have created this problem ourselves by accepting this divisive terminology and using it in everyday discourse. And I asserted that we should abandon, forever, what I think is an artificial and inaccurate distinction.

But such a change raises a potential problem. Support for what we will no longer call basic research has, for quite some time, piggybacked on the support for what we will no longer call translational research, which was what scientific leaders presented to governments and laypeople as the raison d'etre for public support of biomedical research. Generally, they didn't talk much about basic research at all, believing that the public wouldn't understand it very well and therefore wouldn't support it. They understood its importance themselves, so they paid for it, but they didn't advertise it. National Institutes of Health (NIH) director Francis Collins's now-famous remark that "We're not the National Institutes of Basic Sciences" is but one example of this mentality. If we now are to talk about all research using the same language, how do we justify the support of projects that don't have an obvious clinical relevance, and may never have one?

This problem is becoming more acute because we have oversold some big science projects in order to gain the huge financial support they require. The human genome sequencing effort, which was really a basic research project, was presented as a faster route to diagnosis and cures for a host of diseases, although it typically takes decades for research results to lead to clinical advances. Congress and the public, having bought the original sales pitch, are now asking, "So where are the cures?"

Three articles in the 17 March issue of the Journal of the American Medical Association (JAMA) highlight this increasing impatience. They concern the War on Cancer,

*Correspondence: petsko@brandeis.edu

Rosenstiel Basic Medical Sciences Research Center, Brandeis University, Waltham, MA 02454-9110, USA a huge increase in both funding and responsibilities for the US National Cancer Institute (one of the institutes that make up the National Institutes of Health) that was started by President Richard Nixon in 1971. Ignoring the fact that the language of the legislation implied that cancer was one disease, which it most assuredly is not, and therefore should have one cure, which it most assuredly does not, the war has led to $\$ 100$ billion dollars in research funding in the past 40 years, much of which has been spent on 'basic' research in cellular and developmental biology. Now, as Susan Gapstur and Michael Thun point out [1], the cancer war has become a lightning rod, even for some who support its goals. "Frustration about the pace of its progress," they write, "has led some critics to dismiss advances that have been made," and "nearly 1 in 2 men and more than 1 in 3 women will be diagnosed with cancer given the current lifespan." The annual cost to the United States of all cancers, as given by Elena Elkin and Peter Bach in an accompanying article [2], is more than $\$ 90$ billion a year (by comparison, the entire NIH budget is just a little over $\$ 30$ billion). As more families face cancer-associated medical costs that can wipe out a lifetime of savings in a single year, the demand that scientists deliver on their promises is growing from a rumbling to a chorus.

Of course, there have been many successes in the cancer war, most of them resulting from fundamental discoveries about how cell growth is regulated and how cancer starts. Miracle drugs have turned testicular cancer and gastrointestinal stromal cancer and chronic myelogenous leukemia, to name but a few, into treatable diseases in many cases. But there are over 100 different forms of cancer, and most of them still have no cure if the disease is not caught at its earliest stages. Faced with this reality, the instinct of many scientific administrators and researchers is to make even more promises, and to push even harder for more applied research. Writing in the same issue of JAMA, John Niederhuber [3], the current Director of the National Cancer Institute, does exactly that: "To realize a future of personalized medicine, the translation of genomic and functional biology discoveries into clinical practice is essential."

So you see what we're up against. We should talk about research as a seamless whole, a continuum of effort that flows from fundamental discoveries with no obvious 
application inexorably to the prevention and treatment of human diseases. Yet in order to justify it to the public, we have created a distinction that could ultimately tear the biomedical community asunder. How do we make people understand why it is in their best interest for us to do things that have no apparent connection to their concerns?

An old joke encapsulates the problem. A drunkard is looking for his lost car keys at night under a lamppost. A passerby offers to help and asks exactly where he lost them. "Over there," he replies, pointing off into the darkness. "But then, why are you looking for them here?" says the puzzled samaritan. The drunkard explains, "Because the light's better here."

If the only kind of research we do is based on what we already know, we are looking where we already have light. If it turns out that's where the keys are, fine. But we usually aren't sure where the keys are, so we also need to go looking in the darkness. "Basic research" is the light that shines in that dark.

Now, I realize that basing support for all forms of research on a joke may not be the most politically astute of ideas - although I bet it would be a pretty good tactic if you have to explain biomedical research to a gathering of laypeople. Besides, in this age of 10-second sound bites, we need something more immediately memorable, and digestible. But the metaphor of hunting for what is lost provides the answer.

The greatest reassurance we can offer people with lifethreatening or crippling illnesses is that we are leaving no stone unturned in our efforts to find them a treatment. If we only do research that applies discoveries we already have made, we are only looking under stones that have already been turned. That we must do, but if it's all we do, it's not enough. We also need to turn over new stones, because we have no idea where the answers lie. I think anyone can understand that, and appreciate it. This metaphor makes clear the value, and continuity, of all forms of scientific research. And it allows us to discard the 'basic' and 'translational' dichotomy once and for all.

When I go onto the web site of the National Institutes of Health [http://www.nih.gov], which includes the National Institute of General Medical Science (that's 'Basic Sciences', in fact), I notice that this gigantic human endeavor has no motto (it says "The Nation's Medical Research Agency" as a subtitle, but any marketing expert would turn his or her nose up at such a dull and unmemorable phrase). I think it needs one. It should be something that any layperson can immediately grasp, something that speaks to the dedication, commitment, passion, and effort of biomedical scientists to do everything in our power to better their lives. It should be not just NIH's motto, but our motto. What could be better than "No Stone Unturned".

Published: 29 March 2010

References

1. Gapstur SM, Thun MJ: Progress in the war on cancer. JAMA 2010, 303:1084-1085.

2. Elkin EB, Bach PB: Cancer's next frontier: addressing high and increasing costs. JAMA 2010, 303:1086-1087.

3. Niederhuber JE: Translating discovery to patient care. JAMA 2010, 303:1088-1089.

doi:10.1186/gb-2010-11-3-112

Cite this article as: Petsko GA: No stone unturned. Genome Biology 2010, 11:112. 